\title{
DETERMINATION OF ESTERASE D (EsD) GENOTYPE IN CASES WITH TRISOMY 13
}

\author{
Yukio TaKahashi, Kouji Narahara, and Hiroshi Kimoto \\ Department of Pediatrics, Okayama University School of \\ Medicine, Okayama 700, Japan
}

\begin{abstract}
Summary To provide a rationale for gene dosage study of esterase D (EsD) in a case with trisomy 13 where the determination of gene dosage effects is complicated owing to the dimeric structure of EsD and the diversity in catalytic activity between different EsD phenotypes, we assayed red cell EsD activities and analyzed its isozyme patterns using starch gel electrophoresis and isoelectric focusing in five cases with partial trisomy 13 (Cases 1,2 and 3) and full trisomy 13 (Cases 4 and 5). From mean EsD activities of three common EsD phenotypes (1,2-1 and 2) in a total of 197 normal individuals, theoretical values of the total EsD activity and activity ratios of respective EsD isozymes were calculated for each genotype expected in a trisomy case. The observed total EsD activities and activity ratios of respective EsD isozymes, which were measured on starch gel zymograms by a spectrophotometric scanner, in the five cases led us to assume that Case 1 had EsD genotype 1-1-1, Case 2 genotype 2-2-1 and the remaining three cases genotype 2-1-1. The interpretations in three cases (Cases 1, 2 and 4) were further justified by the study of EsD phenotype in their parents. Isoelectric focusing, on the other hand, was insufficient to distinguish between genotypes 2-2-1 and 2-2 or between genotypes 2-1-1 and 2-1. These results suggested that the assay of EsD activity along with analysis of EsD isozyme pattern by starch gel electrophoresis is practically the best procedure to identify EsD genotype in a trisomy individual. Furthermore, this procedure proved to be useful to ascertain the parental origin of nondisjunction in some cases with trisomy 13 .
\end{abstract}

\section{INTRODUCTION}

Esterase D [EsD, EC 3.1.1.1] is an enzyme ubiquitously present in human tissues, of which locus is governed by two common alleles, $E s D^{\prime}$ and $E s D^{2}$, in every 
ethnic group (Hopkinson et al., 1973). Although the biological function of EsD still remains unknown, it is an important gene marker in the context that the locus is closely linked with the retinoblastoma locus in the region of 13q14.1 (Sparkes et al., 1983). As a result, there have been many reports on gene dosage study of EsD in retinoblastoma patients with partial monosomy 13 and cases with partial or full trisomy 13 (Sparkes et al., 1980; Strong et al., 1981; Rivera et al., 1981; Junien et al., 1982; Oishi et al., 1984; Turleau et al., 1983). The determination of gene dosage effects for EsD is simple in cases with partial monosomy 13, whereas that is rather complicated in cases with trisomy 13 because of the dimeric structure of EsD and the fact that one allele $\left(E s D^{2}\right)$ contributes less to the total EsD activity than the other $\left(E s D^{I}\right)$. In most of the previously reported studies concerning trisomy cases, the assessments of gene dosage effects have been based upon the comparisons of EsD activities of patients with those of normal individuals with the same EsD phenotype or mean parental values.

In Japanese, however, the frequency of $E s D^{2}$ is much higher as compared with Caucasians, and the proportion of trisomy cases with heterozygous genotypes containing one or two $E s D^{2}$ alleles is high. Gene dosage studies in such cases, therefore, require not only quantitative but also qualitative assays of EsD. In this paper, we described the results of assays of red cell EsD activities and analyses of EsD isozyme patterns using starch gel electrophoresis and isoelectric focusing in five cases with partial or full trisomy 13. A combination of measurement of EsD activity with analysis of EsD isozyme pattern on starch gels was shown to be practically the best procedure to identify EsD genotypes in trisomy cases.

\section{MATERIALS AND METHODS}

Subjects. The patients consisted of three cases with partial trisomy 13 (Cases 1,2 and 3) and two cases with full trisomy 13 (Cases 4 and 5). Case 1 (Family A III-1 in Fig. 1), the proband, was ascertained because of multiple malformations. Cytogenetic study using G-, $\mathrm{C}$ - and N-banding techniques demonstrated an extra amount of chromosome material on the short arm of chromosome 13 (Fig. 2, a). His father (Family A II-1 in Fig. 1) was found to have a pericentric inversion of one chromosome 13 (Fig. 2, b), and the high-resolution banding analysis of prometaphase cells revealed that the breakpoints had occurred at $13 \mathrm{p} 11.2$ and $13 \mathrm{q} 14.11$ (Fig. 3, b). The abnormal chromosome of Case 1 (Fig. 3, a), in which the segment $13 \mathrm{q} 14.11 \rightarrow$ qter was duplicated and the segment $13 \mathrm{p} 11.2 \rightarrow$ pter was deficient, was thought to be a recombinant resulting from a meiotic crossing over between chromosomes 13 of the father (Fig. 4). The karyotype of Case 1 was designated as 46,XY,rec(13), dup q,inv(13)(p11.2q14.11)pat. Case 2 (Family A III-2 in Fig. 1), a cousin of Case 1, was ascertained to have the same recombinant chromosome as in Case 1, three years and a half after the birth of Case 1. The mother (Family A II-4 in Fig. 1) was shown to be a carrier of the inversion. 


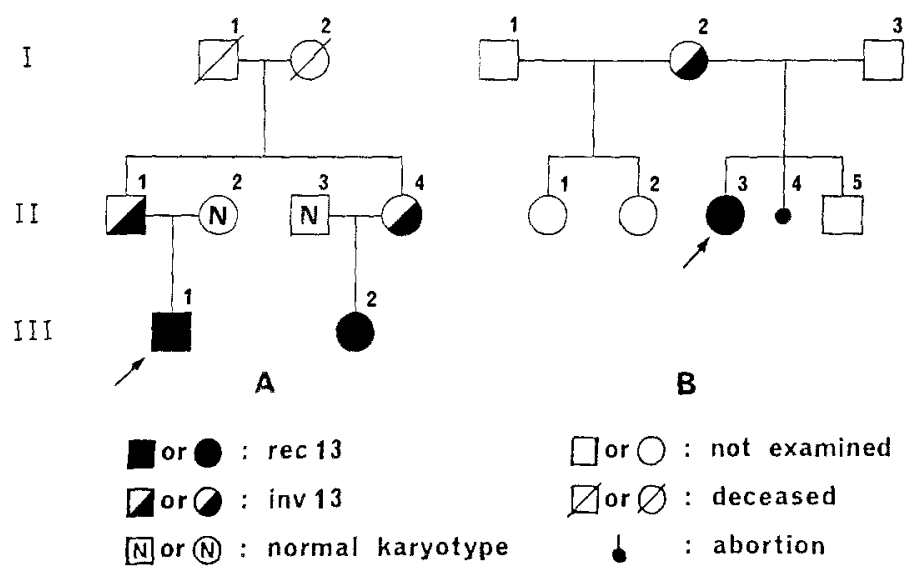

Fig. 1. Pedigrees of the cases with partial trisomy 13. rec(13): recombinant chromosome 13, and inv(13): pericentric inversion of chromosome 13.

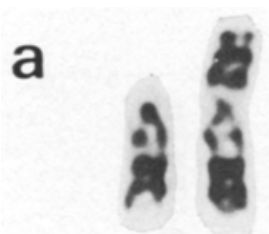

13 rec13

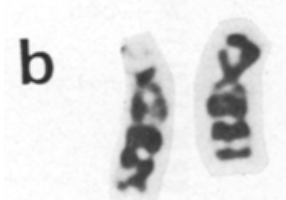

13 inv13

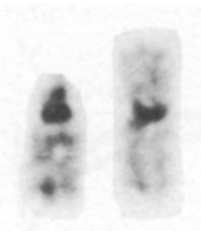

13 rec13

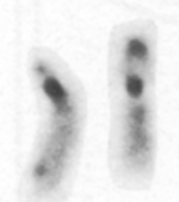

13 inv13

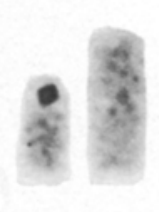

13 rec13

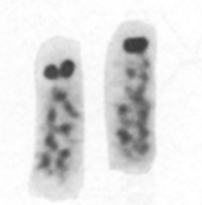

13 inv13

Fig. 2. Partial karyotypes of Case 1 (a) and the father (b). Left, G-banding; middle, C-banding; right, N-banding.

Case 3 (Family B II-3 in Fig. 1) was also a recombinant product of a carrier mother with a pericentric inversion of chromosome 13 (Family B I-2 in Fig. 1), in which the breakpoints were exactly the same as those of the carriers in the family A. There was one early trimester spontaneous abortion after the birth of Case 3. No chromosome analysis of the father (Family B I-3 in Fig. 1) and three other apparently normal siblings (Family B II-1, 2 and 5 in Fig. 1) was available. Since the breakpoints in the two families were identical and the occurrence of this type of chromosome abnormality was restricted to Okayama Prefecture in Japan, they 

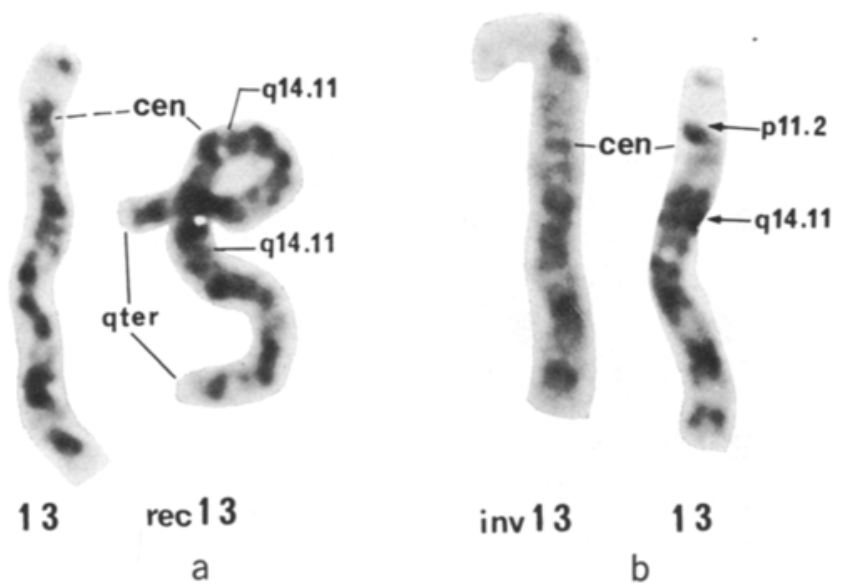

Fig. 3. High-resolution G-banding patterns of chromosomes 13 from Case 2 (a) and the mother (b). Arrows indicate breakpoints and cen denotes centromere.

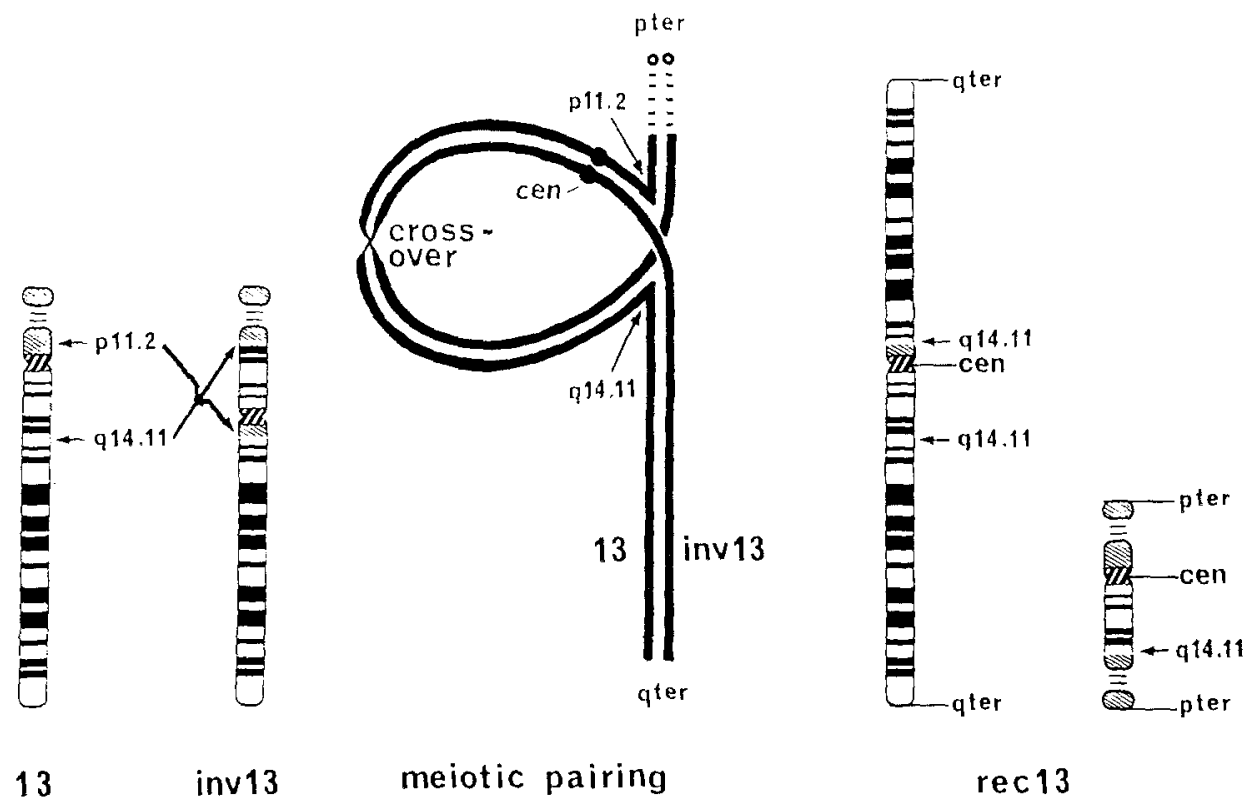

Fig. 4. Schematic representation of the mechanism through which recombinant chromosome 13 (rec13) arise from the pericentric inversion of chromosome 13 (inv13).

seemed to have the common ancestral origin. Clinical findings of the three cases with partial trisomy 13 were similar to those of the full trisomy 13 syndrome except birth weight, gross internal malformations and life prognosis (Table 1).

Cases 4 and 5 were ascertained on the clinical basis to have the trisomy 13 
Table 1. Clinical findings of the three cases with partial trisomy 13 .

\begin{tabular}{|c|c|c|c|c|}
\hline & Case 1 & Case 2 & Case 3 & Full trisomy 13 \\
\hline Birth weight below $2,500 \mathrm{~g}$ & $3,300 \mathrm{~g}$ & $2,580 \mathrm{~g}$ & $2,800 \mathrm{~g}$ & $60-80 \%$ \\
\hline Mental retardation & + & + & + & $90-100 \%$ \\
\hline Microcephaly & - & + & + & $60-90 \%$ \\
\hline Scalp defect & - & - & - & $20-80 \%$ \\
\hline Sloping forehead & - & - & - & $50-100 \%$ \\
\hline Low-set and/or malformed ears & $+1+$ & $+1+$ & $+1+$ & $70-90 \%$ \\
\hline Deafness & + & + & + & $?$ \\
\hline Coloboma iris & - & + & - & $20-60 \%$ \\
\hline Abnormal eyelashes and eyebrows & + & + & + & ? \\
\hline Flat nasal bridge & + & + & + & ? \\
\hline Cleft palate and/or lip & $+1+$ & $+1+$ & $-/-$ & $30-80 \%$ \\
\hline Micrognathia & - & - & - & $40-80 \%$ \\
\hline Capillary hemangiomata & + & + & + & $20-70 \%$ \\
\hline Polydactyly & + & + & + & $60-80 \%$ \\
\hline Cardiac defects & - & - & - & $70-80 \%$ \\
\hline Renal abnormalities & - & Horse-shoe kidney & - & $40-60 \%$ \\
\hline Intestinal malformation & $\ldots$ & - & - & $30-50 \%$ \\
\hline Epileptic seizures & + & + & + & $20-60 \%$ \\
\hline Poor life prognosis & $\begin{array}{c}\text { alive } \\
(6 \mathrm{yr})\end{array}$ & $\begin{array}{c}\text { alive } \\
\text { (3 yr) }\end{array}$ & $\begin{array}{c}\text { alive } \\
(5 \mathrm{yr})\end{array}$ & $100 \%$ \\
\hline
\end{tabular}

syndrome immediately after birth. Chromosome examinations showed regular trisomy 13 in both cases. Cases 4 and 5 died on the second and 14th day of life, respectively. Autopsy revealed various visceral malformations.

Assay of red cell EsD activity. Blood samples were collected using heparin, and hemolysates were prepared according to the method of Beutler (1975). Hemoglobin concentration in hemolysates was determined by the cyanmethemoglobin method. Red cell EsD activity was measured fluorophotometrically with the modified method of Sparkes et al. (1979). After the reaction mixture containing 1.96 $\mathrm{ml}$ of $10 \mathrm{~mm}$ sodium acetate buffer ( $\mathrm{pH} 5.5$ ) and $20 \mu \mathrm{l}$ of $10 \mathrm{~mm}$ 4-methylumbelliferyl acetate was allowed to stand for $5 \mathrm{~min}$ at $37^{\circ} \mathrm{C}$, the reaction was initiated by adding $20 \mu$ of hemolysate diluted $1: 40$ in a $\beta$-mercaptoethanol-EDTA stabilizing solution. The change in fluorescence due to a hydrolysis product, 4-methylumbelliferone, was followed with a fluorescence spectrophotometer (Hitachi 650-10 LC) at $445 \mathrm{~nm}$ with excitation at $320 \mathrm{~nm}$ for $3 \mathrm{~min}$, during which time the reaction was proportional. For the standardization, varying amounts of purified 4-methylumbelliferone were added to the reaction mixture. There was a linear calibration 


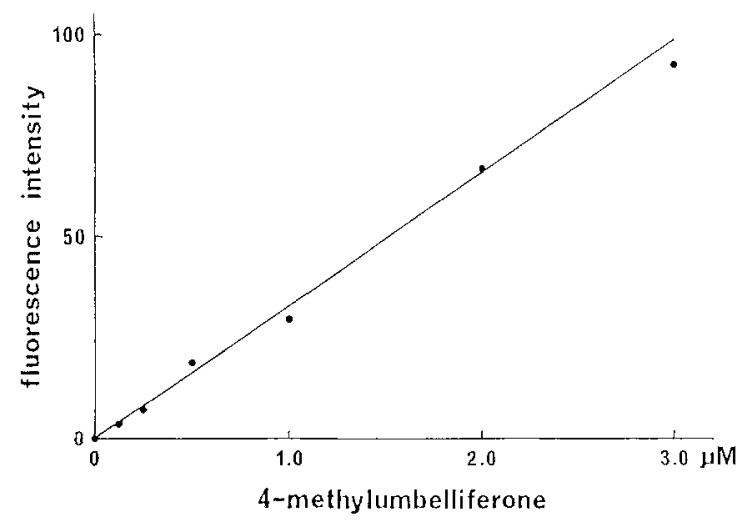

Fig. 5. Standard dose curve for 4-methylumbelliferone. Relative fluorescence intensities were given on the ordinate and concentrations of 4-methylumbelliferone on the abscissa.

curve for 4-methylumbelliferone concentration ranging from 0 to $2.0 \mu \mathrm{M}$ (Fig. 5).

Electrophoresis. Red cell EsD phenotypes were determined using starch gel electrophoresis as described by Harris and Hopkinson (1976). The staining solution was freshly prepared before use with 4-methylumbellifery acetate in a concentration of $0.01 \%(\mathrm{w} / \mathrm{v})$ in $0.1 \mathrm{M}$ phosphate buffer $(\mathrm{pH} 6.5)$. The stain was applied to the cut surface of the gel on a filter-paper overlay, and after a few minutes the gel was photographed under a long-wave UV light using Kodak Tri-X pan films with UV and yellow filters. The fluorescence intensities of respective EsD isozymes were measured on printed photographic papers (Mitsubishi Gekko SP VR-3) with a Shimadzu spectrophotometric scanner at $600 \mathrm{~nm}$ in the refractance mode.

Isoelectric focusing (IEF) was carried out in an electrophoretic chamber, LKB Multiphor 2117 , at $4^{\circ} \mathrm{C}$, according to the method of Yuasa et al. (1985). Polyacrylamide gels $(\mathrm{T}=4.1 \%, \mathrm{C}=4.5 \%)$ were prepared with a dimension of $200 \times 100 \times$ $0.5 \mathrm{~mm}$ and a concentration of an ampholyte (Pharmalyte, $\mathrm{pH} 4.0-6.5$ ) of $4.9 \%$. As anode and cathode solutions, $1 \mathrm{M} \cdot \mathrm{H}_{3} \mathrm{PO}_{4}$ and $0.2 \mathrm{~N} \mathrm{NaOH}$ were employed, respectively. Hemolysates specimens were applied to the gel $2 \mathrm{~cm}$ from the anodal electrode strip, using Toyo No. 1 filter paper wicks, $8 \times 4 \mathrm{~mm}$. After $30 \mathrm{~min}$ prefocusing at a constant power of $3.5 \mathrm{~W}$, a $3 \mathrm{hr}$ focusing was performed at the same power up to $1,200 \mathrm{~V}$ and subsequently at a constant voltage. The $\mathrm{pH}$ gradient of the gel was determined with a surface electrode. The staining was the same as in starch gel electrophoresis.

Assays of EsD activities and electrophoretic studies were performed on the day when the blood samples were collected.

Analysis of $Q$-banding heteromorphism. Using the method of Caspersson et al. (1970), the segregation of QFQ (Q-bands by fluorescence using Quinacrine)-heteromorphism of the paracentromeric region of chromosome 13 was studied in families of the 2 cases with full trisomy 13 . 
Theorem. EsD is thought to be a dimer with a random recombination between two polypeptide subunits, and a heterozygote shows the three-banded pattern consisting of two homomeric and one heteromeric isozymes. The quantity of each isozyme product is the function of combination between the two different polypeptide subunits, of which ratio in synthesis is determined by the gene dosage of each allele. In heterozygous individuals with EsD genotypes 2-1, 2-2-1 and 2-1-1, the quantity of each isozyme product can be expressed as follows:

Isozyme 2-2

Isozyme 2-1

Isozyme 1-1

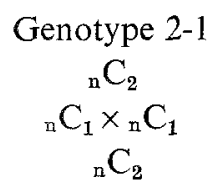

$$
\begin{gathered}
2-1-1 \\
{ }_{n} C_{2} \\
{ }_{n} C_{1} \times 2{ }_{n} C_{1} \\
2{ }_{n} C_{2}
\end{gathered}
$$

When $\mathrm{n}$ is infinitely great, the ratio of each isozyme quantity is $1: 2: 1$ for genotype $2-1,4: 4: 1$ for genotype 2-2-1, and $1: 4: 4$ for genotype $2-1-1$. Given that $\alpha$ is the activity of the homomeric isozyme $2-2, \beta$ the activity of the homomeric isozyme 1-1 and $\gamma$ the total EsD activity in heterozygote 2-1, we can calculate $\delta$, the activity of the heteromeric isozyme 2-1, from the subsequent equation: $\delta=$ $2 \gamma-\frac{1}{2}(\alpha+\beta)$. It follows that the ratios of each isozyme activity in genotypes $2-1$, 2-2-1 and 2-1-1 can be designated to be $\alpha: 2 \delta: \beta, 4 \alpha: 4 \delta: \beta$ and $\alpha: 4 \delta: 4 \beta$, respectively. The total activity will be $\frac{1}{9}(4 \alpha+4 \delta+\beta) \times 1.5$ for genotype $2-2-1$ and $\frac{1}{9}(\alpha+$ $4 \delta+4 \beta) \times 1.5$ for genotype $2-1-1$.

\section{RESULTS}

Distributions of red cell EsD activities in three common EsD phenotypes from a total of 197 normal individuals were shown in Fig. 6. Although there is a wide variation in the enzyme activity within each EsD phenotype, the mean activities differed significantly among the three phenotypes: $4.18 \pm 0.51 \mu \mathrm{m} / \mathrm{min} / \mathrm{g} \mathrm{Hb}$ for EsD phenotype $1(\mathrm{n}=82), 3.34 \pm 0.42 \mu \mathrm{M} / \mathrm{min} / \mathrm{g} \mathrm{Hb}$ for EsD phenotype $2-1(\mathrm{n}=91)$ and $2.34 \pm 0.31 \mu \mathrm{M} / \mathrm{min} / \mathrm{g} \mathrm{Hb}$ for EsD phenotype $2(\mathrm{n}=24)$. Thus, the mean activity of the heteromeric isozyme $2-1$ was calculated to be $3.375 \mu \mathrm{M} / \mathrm{min} / \mathrm{g} \mathrm{Hb}(95 \%$ confidence limits: 3.315 to $3.435 \mu \mathrm{M} / \mathrm{min} / \mathrm{g} \mathrm{Hb}$ ) by the equation described in the theorem. Applying these mean values, theoretical values of the total EsD activities and the activity ratios of respected EsD isozymes were obtained for each genotype expected in a case with trisomy 13 (Table 2).

Starch gel electrophoretic patterns in the five cases with partial or full trisomy 13 were shown in Figs. 7,8 and 9, and the results of spectrophotometric scanning of each EsD isozyme activity together with the assays of total EsD activities in the five cases were given in Table 3. The observed ratios of isozyme distributions in 


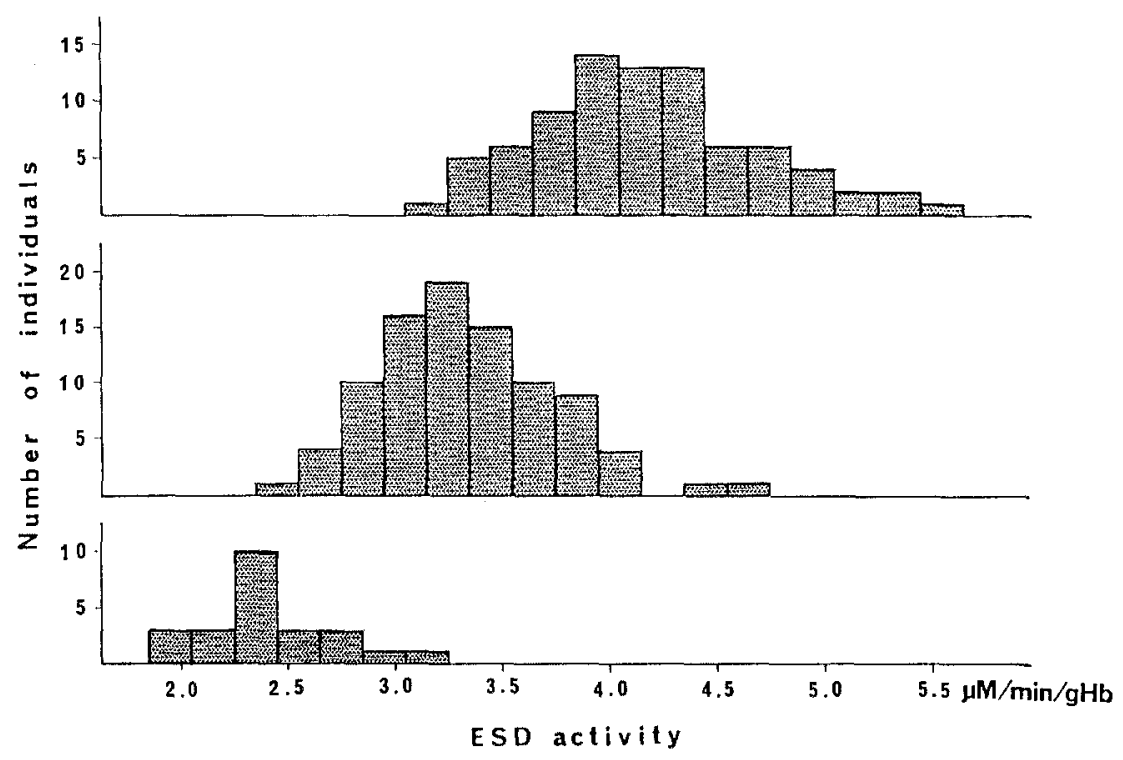

Fig. 6. Distributions of red cell EsD activities in three common EsD phenotypes from a total of 197 normal individuals (from top to bottom, EsD phenotype 1, 2-1 and 2).

Table 2. Expected values of the total EsD activity and the activity ratios of respective EsD isozymes in genotype 2-1 and genotypes supposed in trisomy 13.

\begin{tabular}{rcccccc}
\hline & \multicolumn{5}{c}{ EsD genotype } \\
\cline { 2 - 5 } & $2-1$ & $1-1-1$ & $2-2-1$ & $2-1-1$ & $2-2-2$ \\
\hline $\begin{array}{l}\text { Mean total EsD activity } \\
(\mu \mathrm{M} / \mathrm{min} / \mathrm{g} \mathrm{Hb})\end{array}$ & 3.34 & 6.27 & 4.57 & 5.44 & 3.65 \\
$\begin{array}{r}(95 \% \text { confidence limits }) \\
\text { Activity ratio: }\end{array}$ & $(3.25-3.43)$ & $(6.11-6.41)$ & $(4.42-4.71)$ & $(5.31-5.58)$ & $(3.45-3.84)$ \\
& Isozyme 2-2 & $18.2 \%$ & $0 \%$ & $35.5 \%$ & $7.4 \%$ & $100 \%$ \\
Isozyme 2-1 & $50.5 \%$ & $0 \%$ & $49.3 \%$ & $41.4 \%$ & $0 \%$ \\
Isozyme 1-1 & $31.3 \%$ & $100 \%$ & $15.2 \%$ & $51.2 \%$ & $0 \%$ \\
\hline
\end{tabular}

12 normal individuals with EsD phenotype 2-1 were close to the expected value $\left(\gamma^{2}=0.1\right.$, d.f. $\left.=2, \mathrm{p}=0.95\right)$. The comparisons between the observed and expected ratios of each isozyme activity in the five cases led us to assume that Case 1 had EsD genotype 1-1-1, Case 2 genotype $2-2-1 \quad\left(\chi^{2}=0.41\right.$, d.f. $\left.=2,0.75<p<0.9\right)$, Case 3 genotype $2-1-1\left(\chi^{2}=0.18\right.$, d.f. $\left.=2,0.9<\mathrm{p}<0.95\right)$, Case 4 genotype $2-1-1\left(\chi^{2}=0.44\right.$, d.f. $=2,0.75<p<0.9)$ and Case 5 genotype 2-1-1 $\left(\chi^{2}=0.27\right.$, d.f. $\left.=2,0.75<p<0.9\right)$. The observed values of the total EsD activity were also in agreement with the expected ones in all cases but one (Case 1), where the observed activity was unexpectedly high. The excessive activity in this case may be related to the relatively 


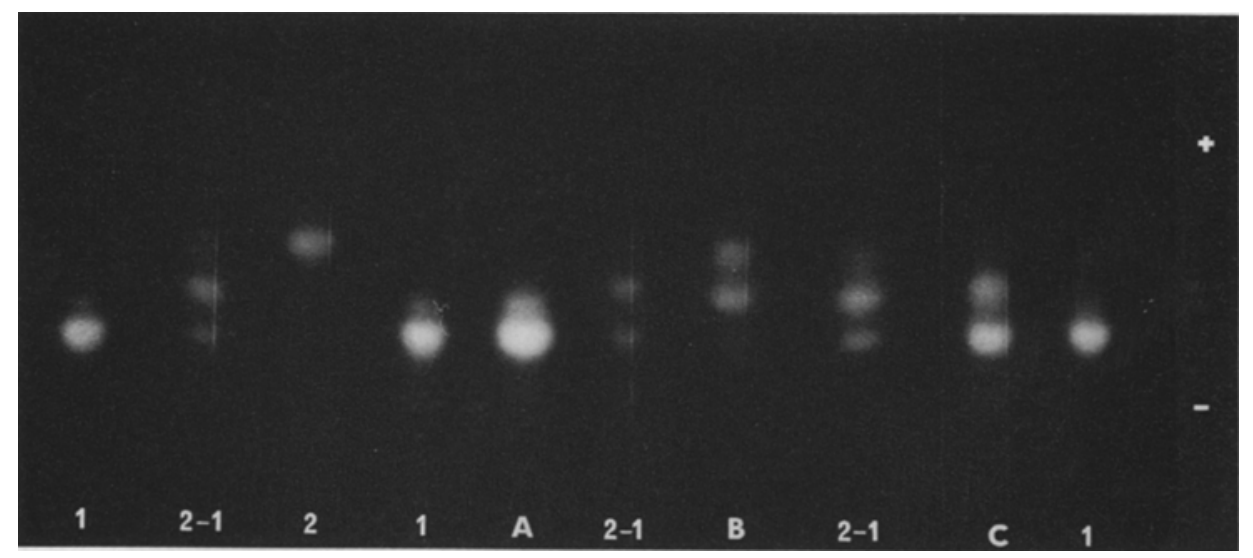

Fig. 7. Starch gel electrophoretic patterns of EsD in the cases with partial trisomy 13. Case 1 (A), Case 2 (B) and Case C (C). In EsD phenotypes 1 (1) and 2 (2) only the homomeric isozyme 1-1 or 2-2 is seen, while in phenotype 2-1 (2-1) the heteromeric isozyme $2-1$ is observed in addition to the two homomeric isozymes.

Table 3. Observed values of the total EsD activity and the activity ratios of respective EsD isozymes in individuals with EsD phenotype 2-1 and the five cases with trisomy 13.

\begin{tabular}{cccrrrrr}
\hline & & $\begin{array}{c}\text { Individuals } \\
\text { with EsD } \\
\text { phenotype 2-1 }\end{array}$ & Case 1 & Case 2 & Case 3 & Case 4 & Case 5 \\
\hline Total EsD activity $(\mu \mathrm{M} / \mathrm{min} / \mathrm{g} \mathrm{Hb})$ & 3.34 & 7.39 & 4.86 & 5.74 & 5.36 & 6.02 \\
Activity ratio: & Isozyme 2-2 & $19.4 \%^{\mathrm{a}}$ & $0 \%$ & $36.4 \%$ & $8.4 \%$ & $10.2 \%$ & $9.4 \%$ \\
& Isozyme 2-1 & $48.4 \%^{\mathrm{a}}$ & $0 \%$ & $51.5 \%$ & $38.7 \%$ & $38.9 \%$ & $41.0 \%$ \\
& Isozyme 1-1 & $32.3 \% \mathrm{a}$ & $100 \%$ & $12.1 \%$ & $52.9 \%$ & $50.9 \%$ & $49.6 \%$
\end{tabular}

Mean values from 12 normal individuals.

high value in one of the parents $(5.12 \mu \mathrm{M} / \mathrm{min} / \mathrm{g} \mathrm{Hb}$ in the mother and $4.37 \mu \mathrm{M} / \mathrm{min} /$ $\mathrm{g} \mathrm{Hb}$ in the father). The interpretations of genotypes in Cases 1 and 2 were verified by the study of EsD phenotype in the parents (in Case 1 both parents had phenotype 1 while in Case 2 the mother had phenotype 2-1 and the father 2).

Furthermore, the segregation study of QFQ-heteromorphism of the paracentrometric region of chromosome 13 in Case 4 disclosed that the extra chromosome $13 \mathrm{had}$ originated from the paternal first meiotic nondisjunction (Fig. 8, A). Therefore, it was conceivable that the case inherited two different $E s D^{\prime}$ alleles from the father and one $E S D^{2}$ allele from the mother, confirming our interpretation of the genotype (Fig. 8, B). In Case 5, on the other hand, the origin of the extra chromosome 13 could not be ascertained by the study of QFQ-heteromorphism, because both parents showed the same QFQ-heteromorphism in one of chromosomes 13 (Fig. 9, A). EsD phenotypes of the parents, however, were informative (the mother 


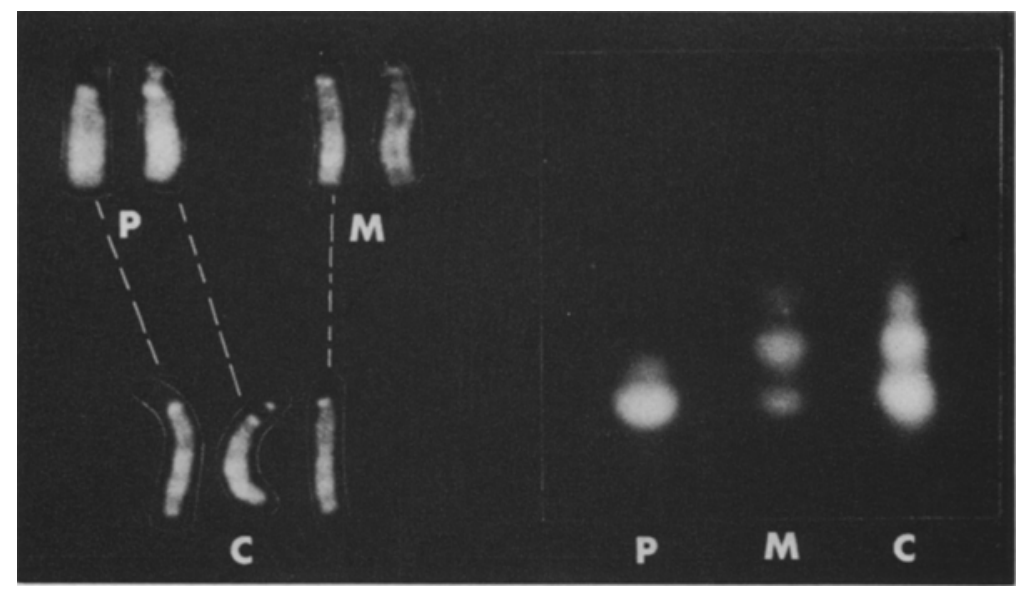

A

B

Fig. 8. A: Q-banding of chromosomes 13 from Case 4 (C), the father (P) and mother (M). B: Starch gel electrophoretic patterns of EsD in Case $4(C)$, the father (P) and mother (M).

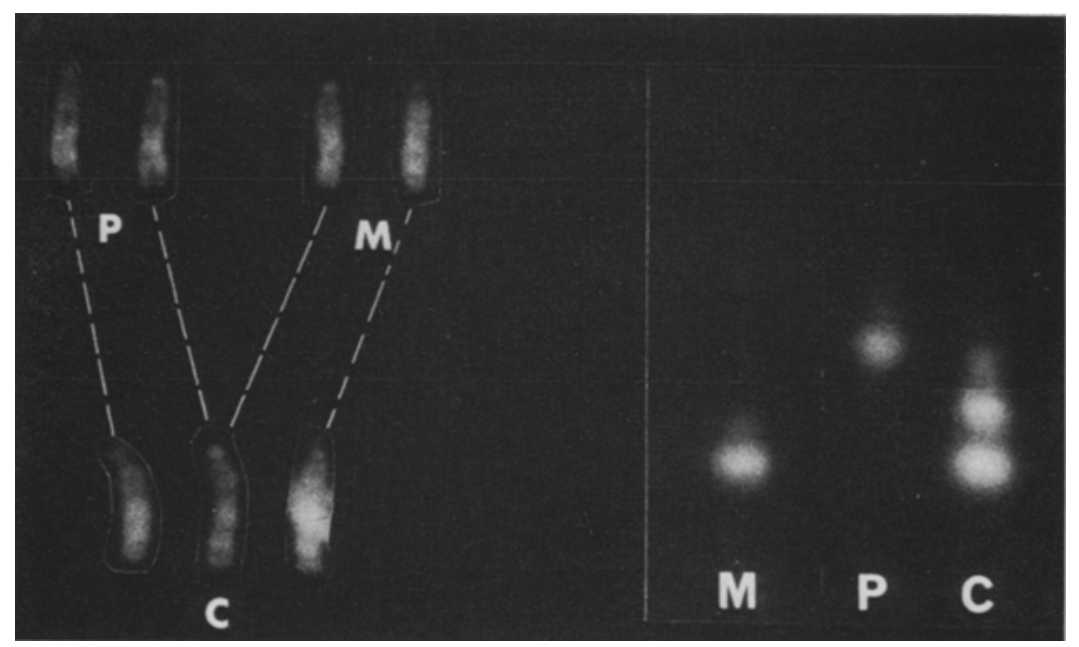

A

B

Fig. 9. A: Q-banding of chromosomes 13 from Case $5(\mathrm{C})$, the father $(\mathrm{P})$ and mother (M). B: Starch gel electrophoretic patterns of EsD in Case $5(C)$, the father (P) and mother (M).

phenotype 1 and the father phenotype 2) (Fig. 9, B). The fact that the case had EsD genotype 2-1-1 suggested the maternal nondisjunction at the first meiotic division.

Lastly, isozyme patterns by IEF in the three patients with partial trisomy 13 


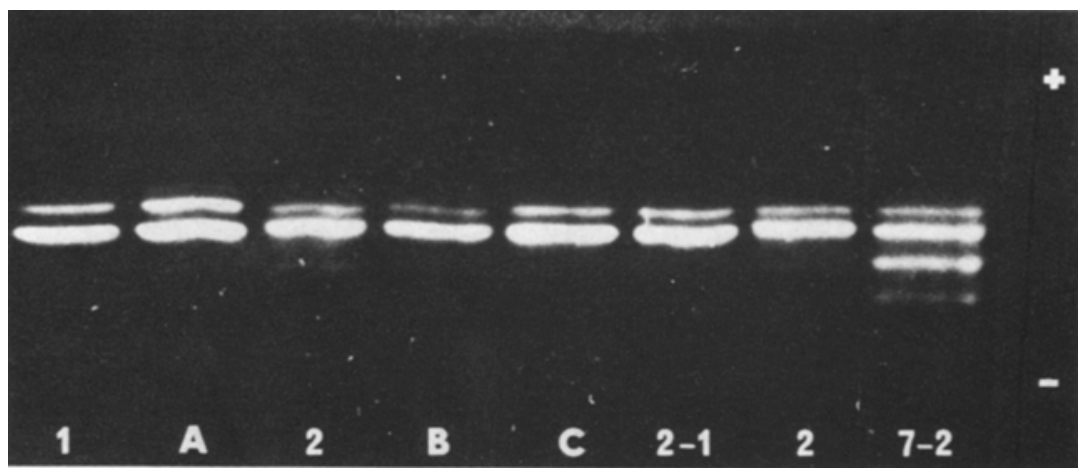

Fig. 10. IEF patterns of EsD in the cases with partial trisomy 13. Case 1 (A), Case 2 (B) and Case $3(\mathrm{C})$. Numerals 1, 2, 2-1 and 7-2 denote EsD phenotypes 1, 2, 2-1 and 7-2, respectively. Major isozyme bands produced by $E s D^{7}$ are much more cathodal than those produced by $E s D^{\prime}$ or $E s D^{2}$.

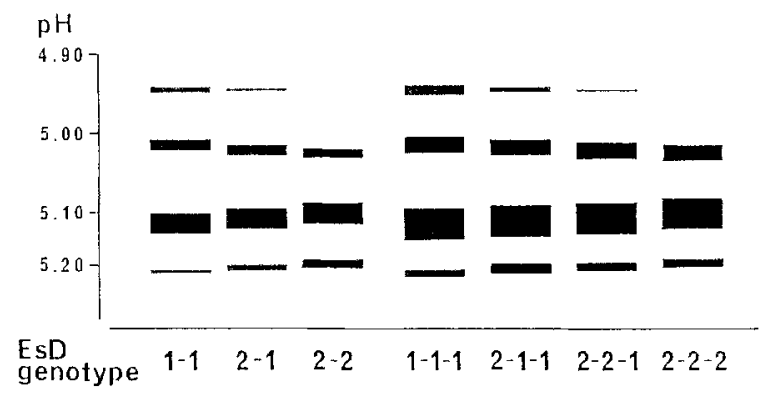

Fig. 11. Diagram showing the localizations of major isozyme bands in EsD genotypes with normal or triplicated common alleles. The $\mathrm{pH}$ gradient is given in the left margin.

were shown in Fig. 10. In none of them, isozyme 7 of which allele exists often in Japanese was observed. Although the localizations of major isozyme bands were somewhat different between the three patients (Fig. 11), it seemed really difficult to distinguish between EsD genotypes 2-1-1 and 2-1 or between EsD genotypes 2-2-1 and 2-2.

\section{DISCUSSION}

The present study had demonstrated that the assay of EsD activity coupled with the analysis of EsD isozyme patterns on starch gel zymograms is practically the best procedure to assess gene dosage effects for EsD in an individual with trisomy 13. The observed total EsD activities and distribution ratios of each EsD isozyme in the five cases with trisomy 13 were in accordance with those expected in the re- 
spective genotypes with triplication of the common EsD alleles, thus verifying our theorem. Although IEF is among the most efficient measures to separate structurally different proteins, the distinction between certain EsD genotypes was not clear.

Nonetheless, the selection of which method is employed in the qualitative assay of EsD should be based on the frequencies of EsD alleles in the population from which a case to be examined is originated. In addition to the common EsD alleles, several rare alleles have been discovered, including Es $D^{3}$ (Bender and Frank, 1974),

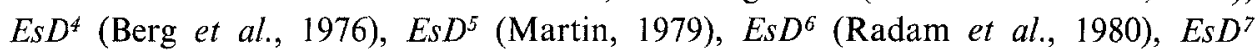
(Nishigaki and Itoh, 1984) and possibly a null allele EsD (Markes et al., 1977). Among them, isozymes produced by $E s D^{5}$ and $E s D^{7}$, that have the similar electrophoretic mobility on starch gels to isozymes produced by $E s D^{2}$ and $E s D^{I}$, respectively, were identified first by IEF. The polymorphic occurrence of $E s D^{5}$ is known to exist in Caucasians, and that of $E s D^{7}$ in Japanese. The frequency of $E s D^{l}$, $E s D^{2}$ and $E S D^{7}$ in Japanese is $0.628,0.364$ and 0.008 , respectively (Nishigaki and Itoh, 1984). Provided that Hardy-Weinberg equilibrium is observed, the frequency of EsD genotype 1-1-1, 2-1-1, 2-2-1 and 2-2-2 in cases with trisomy 13 will be 0.248 , $0.431,0.250$ and 0.048 , respectively. The proportion of genotypes containing one or more $E s D^{7}$ alleles, on the other hand, sums only up to 0.024 . This suggests that the use of starch gel electrophoresis alone may suffice to determine EsD genotypes in the vast majority of Japanese cases with trisomy 13.

The gene frequency of $E s D^{2}$ as well as $E s D^{7}$ are much higher in Japanese than in Caucasians, and accordingly a large part of individuals $(47.3 \%)$ are heterozygous for EsD. The study of EsD genotypes in families of a case with trisomy 13 is sometimes useful to identify the parental origin of the extra chromosome. Since the isozyme product of $E s D^{7}$ cannot be separated from that of $E s D^{1}$ by starch gel electrophoresis, matings of individuals possessing $E s D^{7}$ allele (a total of $3.1 \%$ in all matings) are excluded from the following estimations. In the case of the same genotype in both parents (38.2\% of all matings), children are not informative. On the other hand, where each parent is a different homozygote as in Case 5 of our study (10.5\% of all matings), the origin of nondisjunction can be ascertained in all families. There is another mating as in Case 4, where one parent is homozygous and the other heterozygous ( $48.2 \%$ of all matings). Here, the parental origin can be determined, only when a chromosome bearing an allele that is not present in the other partner missegregates at the second meiotic division. As it has been reported that a third of cases with trisomy 13 have resulted from nondisjunction at the second meiotic division (Ishikiriyama and Niikawa, 1984), the likelihood of such an event is calculated to be $4.0 \%$.

The single use of starch gel electrophoresis in determining EsD genotypes, therefore, allows us to assign the parental origin of nondisjunction in overall $14.5 \%$ of cases with trisomy 13. It will, however, give more informations, if coupled with the analysis of QFQ-heteromorphism in the paracentromeric region of chromosome 
13. When the nondisjunction proves to have occurred at the first meiotic division in one homozygous parent mated with the other heterozygous one, certain genotypes of children (genotype 1-1-1 or 2-2-2) will make the ascertainment possible in further $8.0 \%$ of all matings.

It is notorious that values of red cell EsD activities differ strikingly from one laboratory to the other despite of the use of essentially the same fluorometric method. Our values are roughly 40-60 times as much as those of Sparkes et al. (1979) and ten times higher than those of Horai and Matsunaga (1984). These discrepancies are difficult to explain, but seem to be due in part to the different standardizations. To establish the standard dose curve, we added 4-methylumbelliferone to the reaction mixture containing the substrate 4-methylumbelliferyl acetate in a final concentration of $0.1 \mathrm{~mm}$, because the substrate in such a high concentration at acid $\mathrm{pH}$ shows unnegligible fluorescence and interfere the accurate measurement of the hydrolysis product. The values of EsD activities in our study are similar to those obtained in the spectrophotometric assay (Nishigaki et al., 1983), though the variation of the activity in each EsD phenotype is greater in our study. The further studies using simultaneously the fluorometric and spectrophotometric methods on the same 33 individuals have revealed that there is a good correlation between values measured by the two different methods $(\mathrm{Y}=1.16 \mathrm{X}-0.82, r=0.875$, $\mathrm{p}<0.001$ ) (Fig. 12). This may support the correctness of our standardization procedure.

Finally, triplex gene dosage effects for EsD has been demonstrated in the three cases with trisomy for $13 q 14.11 \rightarrow$ qter. This is in accordance with the recent gene mapping data suggesting that the loci for EsD and retinoblastoma have been assigned to $13 \mathrm{q} 14.1$. The regional mapping at the level of sub-bands has been reported only

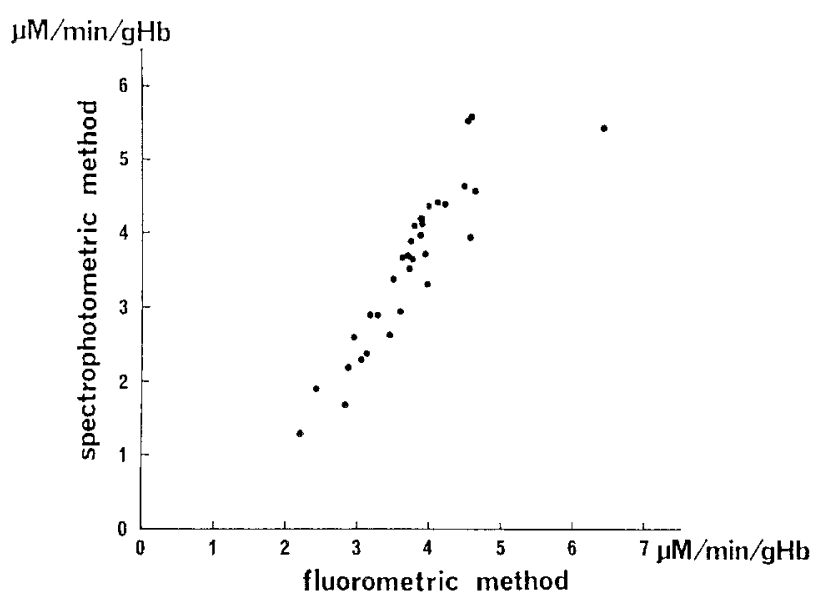

Fig. 12. Correlation between values of red cell EsD activities measured by the fluorometric and spectrophotometric methods on the same 33 individuals. 
by Ward et al. (1984). Their case with retinoblastoma, being monosomic for $13 \mathrm{q} 12 \rightarrow \mathrm{q} 14.11$, was found to have a $50 \%$ EsD activity of normal individuals with the same EsD phenotype. In conjunction with this report, we would like to propose that the EsD locus may lie within the distal portion of the band $13 \mathrm{q} 14.11$. The confirmation of this hypothesis, however, will have to await further gene dosage studies of EsD in cases with partial monosomy or trisomy 13 in which the breakpoints occur within the region q14.1.

\section{REFERENCES}

Berg, K., Schwarzfischer, F., and Wischerath, H. 1976. Esterase D polymorphism; description of the "new" allele EsD'. Hum. Genet. 32: 81-83.

Bender, K. and Frank, R. 1974. Esterase D-Polymorphismus: Darstellung in der Hochspannungselektrophorese und Mitteilung von Allelhäufigkeiten. Humangenetik 23: 315-318.

Beutler, E. 1975. Red Cell Metabolism: A Manual of Biochemical Methods, 2nd Ed., Grune and Stratton, New York, pp. 8-11.

Caspersson, T., Zech, L., Johansson, C., and Modest, E.J. $1970 . \quad$ Identification of human chromosomes by DNA-binidng fluorescent agents. Chromosoma 30: 215-227.

Harris, H. and Hopkinson, D.A. 1976. Handbook of Enzyme Electrophoresis in Human Genetics, North Holland Publishing Company, Amsterdam.

Hopkinson, D.A., Mestriner, M.A., Cortner, J., and Harris, H. 1973. Esterase D: a new human polymorphism. Ann. Hum. Genet. 37: 119-137.

Horai, S. and Matsunaga, E. 1984. Differential enzyme activities in human esterase D phenotypes. Hum. Genet. 66: 168-170.

Ishikiriyama, S. and Nikawa, N. 1984. Origin of extra chromosome in Patau syndrome. Hum. Genet. 68: 266-268.

Junien, C., Despoisse, S., Turleau, C., Nicolas, H., Picard, F., Le Marec, B., Kaplan, J.C., and Grouchy, J. de. 1982. Retinoblastoma, deletion 13q14, and esterase D: application of gene dosage effect to prenatal diagnosis. Cancer Genet. Cytogenet. 6: 281-287.

Markes, M.P., Jenkins, T., and Nurse, G.T. 1977. The red cell glutamic-pyruvic transaminase, carbonic anhydrase I and II and esterase D polymorphisms in the Ambo populations of South West Africa with evidence for the existence of an Es $D^{0}$ allele. Hum. Genet. 37: 49-54.

Martin, W. 1979. Neue Elektrophoresemethoden zur Darstellung von Serum- und Enzympolymorphismen: technische Verbesserungen, Hinweis auf weiteres EsD-Allel. Ärztl. Lab. 25: 6567.

Nishigaki, I., Itoh, T., and Ogasawara, N. 1983. Quantitative variations in polymorphic types of human red cell esterase D. Arm. Hum. Genter. 47: 187-192.

Nishigaki, I. and Itoh, T. 1984. Isoelectric focusing studies of human red cell esterase D: evidence for polymorphic occurrence of a new allele $E s D^{7}$ in Japanese. Hum. Genet. 66: 92-95.

Oishi, H., Nishigaki, I., Futamura, M., and Takebe, H. 1984. Regional assignment of esterase D locus to human chromosome $13 \mathrm{q} 13 \rightarrow$ qter. Cytogenet. Cell Genet. 37: 558-559.

Radam, G., Strauch, H., and Martin, W. 1980. Der Phänotype "Rügen" im Polymorphismus der Esterase D: Hinweis auf die Existenz eines neuen Allels (EsD $D^{6}$. Blut 40:337-341.

Rivera, H., Turleau, C., Grouchy, J. de, Junien, C., Despoisse, S., and Zucker, J.M. 1981. Retinoblastoma-del(13q14): report of two patients, one with a trisomic sib due to maternal insertion. Gene-dosage effect for esterase D. Hum. Genet. 59: 211-214.

Sparkes, R.S., Targum, S., Gershon, E., Sensabaugh, G.F., Sparkes, M.C., and Crist, M. 1979. Evidence for a null allele at the esterase D [EC 3.1.1.1] locus. Hum. Genet. 46: 319-323. 
Sparkes, R.S., Sparkes, M.C., Wilson, M.G., Towner, J.W., Benedict, W., Murphree, A.L., and Yunis, J.J. 1980. Regional assignment of genes for human esterase D and retinoblastoma to chromosome band 13q14. Science 208: 1042-1044.

Sparkes, R.S., Murphree, A.L., Lingua, R.W., Sparkes, M.C., Field, L.L., Funderburk, S.J., and Benedict, W.F. 1983. Gene for hereditary retinoblastoma assigned to human chromosome 13 by linkage to esterase D. Science 219: 971-973.

Strong, L.C., Riccardi, V.M., Ferrell, R.E., and Sparkes, R.S. 1981. Familial retinoblastoma and chromosome 13 deletion transmitted via an insertional translocation. Science 213: 1501-1503.

Turleau, C., Grouchy, J. de, Chavin-Colin, F., Despoisses, S., and Leblanc, A. 1983. Two cases of del(13q)-retinoblastoma and two cases of partial trisomy due to a familial insertion. Ann. Genet. 26: 158-160.

Ward, P., Packman, S., Loughman, W., Sparkes, M., Sparkes, R., McMahon, A., Gregory, T., and Ablin, A. 1984. Location of the retinoblastoma susceptibility gene(s) and the human esterase D locus. J. Med. Genet. 21: 92-95.

Yuasa, I., Tamaki, N., Suenaga, K., and Ito, K. 1985. Low voltage isoelectric focusing for phenotypes of esterase D. Jpn. J. Human Genet. (abstract) 30: 106. 